\title{
28 Research Square \\ Clinical Features of Actinomycosis: A 20-year Experience of A Single Institute in Southern China
}

\section{Yu-yuan Xu}

State Key Laboratory of Organ Failure Research, Guangdong Provincial Key Laboratory of Viral Hepatitis Research, Department of Hepatology Unit and Infectious Diseases, Nanfang Hospital, Southern Medical University

\section{Jian Zhang}

Department of Hepatology Unit and Infectious Diseasea, Nanfang Hospital

\section{Ai-li Lu}

Department of Hepatology Unit and Infectious Diseases, Nanfang Hospital, Southern Medical University Jie Peng

Department of Hepatology Unit and Infectious Diseases, Nanfang Hospital, Southern Medical University Xu-wen Xu ( $\nabla$ xuxuwen95@126.com)

\section{Research article}

Keywords: Actinomycosis; Differential diagnosis; Treatment; Prognosis

Posted Date: July 31st, 2019

DOI: https://doi.org/10.21203/rs.2.12223/v1

License: (1) (i) This work is licensed under a Creative Commons Attribution 4.0 International License.

Read Full License

Version of Record: A version of this preprint was published at Journal of Medical Mycology on September 1st, 2021. See the published version at https://doi.org/10.1016/j.mycmed.2021.101169. 


\section{Abstract}

Background Actinomycosis is a rare indolent infectious disease with nonspecific clinical presentations that delay diagnosis. Although actinomycosis is thought to be more prevalent in developing countries, data from developing countries are scanty. This study was to reveal the current situation of actinomycosis in developing countries and the difference from that in developed countries. Methods Patients fulfilling the inclusion criteria for actinomycosis from Nanfang Hospital in southern China between January 2003 and December 2018 were retrospectively analyzed. We described clinical characteristics, diagnostic procedures, differential diagnosis, and management of actinomycosis of clinical significance. Results Thirty-one patients were included in this study. Disease was diagnosed predominately in the orocervicofacial $(n=14)$, cardiothoracic $(n=11)$, abdominopelvic $(n=5)$, soft tissue $(n=1)$. Diagnosis was obtained from either histopathology $(n=29,94 \%)$ or microbiology $(n=2,6 \%)$. Only one-thirds of patients presented with general symptoms such as fever, weight loss. Ten was lost to follow-up, and median duration of antibiotics was 93.5 days (interquartile range $28-300$ ), whereas the median follow-up time was 34 months (interquartile range 9-132). Ten patients receiving complete resection of the lesion were cured without postoperative use of antibiotic. Only one patients relapsed during the follow-up period. Conclusions Actinomycosis is a rare disease even in developing countries, and both misdiagnosis and missed diagnosis are common. Diagnosis was often delayed and obtained from histopathology postoperatively in developing countries. Hence, clinicians should be aware of this disease in patients with high riks factors. In the future, specific molecular methods may help to improve early diagnosis and treatment.

\section{Background}

Actinomycosis is a rare, chronic, and slowly progressive granulomatous disease caused by filamentous Gram positive anaerobic bacteria from the Actinomycetaceae family[1]. Actinomyces are commensals of the human oropharynx, gastrointestinal tract, and urogenital tract. When tissue integrity is breached(e.g. trauma, radiotherapy, inflammatory perforation) through a mucosal lesion they can invade local structures and organs and become pathogenic[2]. All the tissues and organs may be infected, but four main clinical types of infection can be distinguished, depending on the primary site of infection: orocervicofacial, cardiothoracic, abdominopelvic, and disseminated disease[3]. Actinomycosis is frequently underdiagnosed because of its clinical and imagiological mimicry of other infectious diseases (e.g. nocardiosis, tuberculosis) and malignancies[4]. Although the prognosis of these infections normally is good with medical and surgical treatments, actinomycosis still can lead to the death of patients due to the difficulties of early diagnosis and the severe infection diffusion of Actinomyces[5].

More than 30 species of Actinomyces have been described. The most common pathogenic species is Actinomyces israelii[6]. The mechanisms of pathogenicity of Actinomyces are not completely understood, but the invasion of breached or necrotic tissue has been proposed to be the initiating event for Actinomyces to penetrate and proliferate in deeper body structures[7]. Immunosuppression seems to be 
an important risk factor in the development of infection, as it has been described in patients with human immunodeficiency virus (HIV) infection, leukemia, solid organ malignancies, and others.

Based on the localization of the infection, distinct clinical features are reported, but misleading forms are still frequent. Orocervicofacial actinomycosis is the most common clinical manifestation and it usually follows dental manipulation or trauma to the mouth, although it can arise spontaneously in patients with poor dental hygiene. Patients frequently present with chronic soft tissue swelling and sometimes can also present with abscesses, woody fibrosis, and sinus discharge of sulfur granules[8]. As to abdominopelvic actinomycosis, more than $90 \%$ of cases are diagnosed postoperatively[1]. Most patients may present with non-specific symptoms such as fever, weight loss, and abdominal pain. Erosion through the mucosal barrier caused by perforation of appendicitis, gastrointestinal perforation, previous surgery, neoplasia, and foreign bodies in the gastrointestinal tract or genitourinary tract is considered the possible predisposing factors[9]. Although abdominal disease can spread directly into the pelvis, pelvic actinomycosis is predominantly associated with intrauterine contraceptive devices. Thoracic actinomycosis is mainly caused by the aspiration of oropharyngeal or gastrointestinal secretions into the respiratory tract, although it can sometimes occur as a result of haematogenous seeding, lymphatic spread or spread from the cervicofacial area through the mediastinum[10]. Pulmonary actinomycosis associated with intratracheal or endobronchial foreign bodies have also been reported. The clinical manifestations and imaging characteristics of pulmonary actinomycosis lack specificity, and can lead to confusion with tuberculosis and lung cancer. This may lead to misdiagnosis of the disease, and hence, incorrect treatment[11]. Cases of musculoskeletal actinomycosis, such as that of the long bones are rare, and are typically described as chronic sclerotic or spherical osteomyelitis[12]. Such infections originate from infected adjacent tissues or, less frequently, following hematogenous dissemination. Infections of the urogenital tract[13], eye[14], skin[15], and disseminated disease[3] focalized in the central nervous system (CNS)[16] are rare; a case of penile actinomycosis has also been reported[17]. Actinomyces spp are very susceptible to a wide variety of antimicrobials, thus relatively few doses may render cultures negative. Diagnosis may be difficult because of a general lack of familiarity with the disease and a low success rate in culturing the organism as a result of its fastidious nature.

In our study, almost all the cases are not suspected at the clinical onset of the symptoms. Failure to diagnose the disease in a timely and reliable way seems to increase mortality and unnecessary costly diagnostic investigations. The aim of this study is to highlight the various clinical forms of actinomycosis to help clinician diagnose and treat the disease earlier. In this retrospective study, we report a series of 31 patients with different clinical presentations in a single institute.

\section{Method}

Patients with the diagnosis of actinomycosis at Nanfang Hospital from January 1999 to December 2018 were searched, and those fulfilling the following inclusion criteria were included in this study: microbiological or histological confirmation of actinomycosis from tissue biopsies, and sputum or blood sample specimens. Histological confirmation demonstrated a filamentous branching organism with or 
without sulfur granules. Microbiological confirmation was definded as samples culture-positive for Actinomyces species.

Exclusion criteria were: cases including positive microbiological identification of Actinomyces spp. from superficial site without clinical or radiological hallmarks of infection, and medical records without sufficient data. For each case, we reviewed demographic characteristics, underlying diseases, clinical and radiological manifestations, microbiological results, histological assessment, and treatment and outcome data. Because of the restricted number of individuals included in the study, the quantitative variables were described as medians and interquartile ranges. Categorical data were described as numbers and percentages.

\section{Results}

A total of 31 patients were included in this study. The male:female ratio was 16:15. The median age at diagnosis was 53 years (interquartile range [IQR] 44-61.5). Patients' characteristics are summarized in Table 1. A local risk factor was found in 26 patients (84\%), mainly local tissue injury including dental procedure or poor dentition, surgery, trauma, radiotherapy or intrauterine devices (IUDs). Common risk factors were present in 13 patients, including diabetes mellitus in 3 patients (10\%), immunosuppressive agent intake in 1 patient, malignancies in 6 patients (19\%) previously treated by chemotherapy (5 patients) and radiotherapy (2 patient), smoking in 5 patients and 1 patient with alcoholism. None of the patients were HIV-positive.

Concerning general symptoms, fever was observed in 5 patients (16\%), whereas weight loss and anorexia were present in $8(26 \%)$ and $6(19 \%)$ patients, respectively. Leukocytosis and C-reactive protein elevation were observed in 8 patients $(26 \%)$ and 12 patients (39\%), respectively. Median diagnosis time frame from first symptoms was 150 days (IQR 14-1460).

With respect to anatomical location of infection, orocervicofacial location was the most frequent with 14 patients (45\%). Five patients (patients 8, 10,11, 13 and 14) had maxillary or mandibular osteomyelitis. Patients 13 and 14 both had a local radiotherapy history for nasopharynx and gingival carcinoma respectively. Mandibular osteonecrosis was seen in patient 10 who had a history of radiotherapy and surgery for a gingival cancer. Patient 8 with a bad dental hygiene presented with gingival neoplasm and dentoosteitis. Patient 11 also had maxillary sinus abscess in spite of osteonecrosis. Patient 12 with a history of immunosuppressor intake for rheumatoid arthritis was diagnosed with maxillofacial space infection and had incision and drainage for abscess 1 week before admitted. Patient 2 and 3 both complained about throat discomfort and was confirmed infection after tonsillectomy. Patient 4 underwent a surgery of maxillary ameloblastoma and got postoperative infection which spread to the whole right maxillofacial region. Patient 1, 5, 6, 7, 9 presented with superficial soft tissue masses located in nasal cavity, epiglottis, oropharynx, dorsum of tongue, cheek respectively and was cured after complete resection except for Patient 6 who just underwent diagnostic biopsy following a course of antibiotics. 
Among thoracic forms, all the cases were pulmonary form and presented with respiratory symptom. Cough was the most common presentation, followed by sputum production and hemoptysis. Fever, short of breath and chest pain were also occasionally noted. Patient 23 presented with pulmonary abscess, surgically removed through video-assisted-thoracic-surgery. Patient 16, 21, 24, 25 underwent pneumectomy to exclude tumor. Patient 18 was diagnosed actinomycosis by ThinPrep cytology and finally confirmed lung adenocarcinoma through endobronchial ultrasound guided tranbronchial needle aspiration (EBUS). Patient 15 presented with ground-glass nodules in the inferior lobe of right lung and was considered infection after a consultation of multi-disciplinary team because of low standard uptake

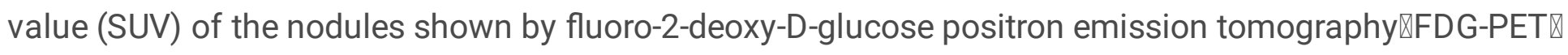
examination. Patient 17 presented with a huge pulmonary occupying lesions, who was just willing to accept fibrobronchoscopy and refused further examination and biopsy. Patient 22 had a history of respiratory failure supported by ventilator for nearly six months secondary to a surgery of calculus of bile duct ten years ago, which led to tracheo-esophageal fistula and tracheal stenosis resolved by tracheal stent implantation, and found necrotic tissue with Actinomyces spp. colonies around the stent. Patient 19, 20 presented with pneumonia and were diagnosed by actinomycosis culture of bronchoalveolar lavage fluid.

Among patients with abdominopelvic localization, an IUD was present in all femal patients. Only Patient 28 presented with systemic symptoms (fever, weight loss) while Patient 26 presented with vaginal bleeding and was diagnosed by diagnostic D\&C (dilatation and curettage). Hematuresis was present in Patient 27 and she was suspected ovarian cancer by accidently through computed tomography (CT) scan. As to the other two male patients, Patient 29 was infected after the ileostomy because of colorectal cancer. Patient 30 was an unexplained case whose infection was located at the abdominal wall of no obvious risk factors and was cured just after surgery. Patient 31 with a clinical history of trauma of right knee 10 years earlier and no other co-morbidities, presented with a persistent fistula and pus for more than 1 year.

The diagnosis of actinomycosis was not suspected on admission for all of cases. The most frequent initial diagnosis suspected were malignancies and widespread infections such as tuberculosis (Table 1). Imaging was performed in $81 \%$ of patients, including ultrasound (3/31[10\%]), X-ray(3/31[10\%]), CT-scan $(15 / 31[48 \%])$, magnetic resonance imaging (MRI) $(3 / 31$ [10\%]) and FDG-PET( $n=6)$. The maximum standardized uptake value of FDG-PET ranged from 2.2 to 7.9. According to FDG-PET results, 3 patients were considered to have malignancy, while the other 3 patients were considered to have inflammation.

In spite of our best effort, 10 patients were still lost to follow-up. In the remaining 21 patients, 11 patients diagnosed by surgery did not take any medicine after surgery in view of the complete resection of the lesion, and all of them were stable and uneventful. The remaining 10 patients were treated with antibiotics, mainly beta-lactams and especially amoxicillin except for Patient 20 who was allergic to penicillin. Median follow-up time was 34 months (9-132). The median duration of treatment was 93.5 days (28-300). The outcome was favorable in 20/21 (95\%) patients except for patient 31 who relapsed during the course of antibiotics. 


\section{Discussion}

In our study, we have described 31 cases of miscellaneous forms of actinomycosis, a rare life-threatening infectious disease with nonspecific features, and as far as we know, this is the largest study of actinomycosis in a single institution in China. In all of the presented cases of actinomycosis it is clear that a late diagnosis of this infection is still an ongoing difficulty, delaying appropriate treatment and increasing infection duration. Although it is readily treatable and curable if the patient is appropriately managed. Thus, this infection represents a great diagnostic challenge.

Actinomycosis is certainly under-reported as a consequence of diagnostic errors, difficulties in confirming the disease, and the empirical utilization of antibiotics. Data about the incidence estimates are nor recent and lack in developing countries. It was reported about one per 300000 in the 1970 s of Cleveland, USA while it was estimated to be one per million in the 1960s of Germany and the Netherlands[1]. The incidence of all forms of actinomycosis is thought to have declined in recent years, especially in developed countries as a result of better oral hygiene and susceptibility to a broad range of antibiotics. Largest series focused on single anatomical forms $[10,18]$, whereas some case reports of unusual presentations have been published in China[3,19]. To our knowledge, there is no report about multiple forms of actinomycosis in China. This study try to outline the etiology, diagnosis, clinical features, treatment, and prognosis of the disease in a teaching hospital in southern China during the past 20 years.

Clinical picture of the actinomycosis can mimic different diseases, for example, tumors, tuberculosis, nocardiosis, fungal or other diseases, consequently, diagnosis may be very difficult[1]. Besides, the bacteriological identification of Actinomyces occur in only a minority of cases because of previous antibiotic therapy, inhibition of Actinomyces growth by concomitant and/or contaminant microorganisms, inadequate culture conditions, or inadequate short-term incubation, especially in developing country[7]. A Gram stain of the specimen is usually more sensitive than culture, especially if the patient had received antibiotics. Actinomyces are non-spore-forming Gram-positive rods. Except for A. meyeri, which is small and nonbranching, all the other species are branching filamentous rods. Sulphur granules are colonies of organisms that appear as round or oval basophilic masses with eosinophilic terminal "clubs" on staining with haematoxylin-eosin. Although the presence of sulphur granules is helpful in making the diagnosis, they are not always recovered in culture confirmed cases of actinomycosis. Only one patient (Patient 16) presented with Sulphur granules in lung specimens. Gram staining of Nocardia spp. is morphologically similar to that of Actinomyces spp. and there is some features that may help to distinguish these 2 microorganisms [20]. In our study, only two patients (Patient 19,20 ) were diagnosed by sputum culture while the rest patients were confirmed by different tissue samples histologically. The failure rate of culture seems higher than that reported in the west, meanwhile the median duration from first symptoms to diagnosis is also longer [21].

Most commonly, the actinomycosis presents as a slowly progressive, indolent infiltration with dense fibrosis, multiple abscesses, fistulas and draining sinuses. Rarely, the infection is acute and rapidly progressive, which is consistent with our study. To date, multiple different clinical features of 
actinomycosis have been described, as various anatomical sites (such as face, bone and joint, respiratory tract, genitourinary tract, digestive tract, central nervous system, skin, and soft tissue structures) can be affected. Our study try to picture the panorama of actinomycosis in multiple possible focalizations.

Actinomyces are commensals of the human oropharynx, and are particularly prevalent within gingival crevices, tonsillar crypts, periodontal pockets and dental plaques, as well as on carious teeth. If the anatomical barriers are breached, the bacteria can become pathogenic and and usually presents as a chronic, painless or occasionally painful soft-tissue swelling of the submandibular or perimandibular region, draining sinus tracts with sulfur granules, difficulties in chewing and chronic/relapsing course of the infection. Consequently, cervicofacial actinomycosis is the most frequent clinical form of actinomycosis, representing approximately $60 \%$ of all reported cases[1]. It is about $45 \%$ in our study, and the susceptibility to a broad range of antibiotics and misdiagnose may account for less cases despite the poor oral hygiene in the most patients. Cervicofacial actinomycosis could be associated with large abscesses and/or mandibular osteomyelitis with or without sinus tract. Risk factors for cervicofacial and oral actinomycosis are dental procedures such as dental extractions, dental caries, trauma, gingivitis, chronic tonsillitis, periodontal disease, otitis or mastoiditis, possibly diabetes and immunosuppression, malnutrition, and local tissue injuries by tumors, surgery, or irradiation. All the patients were identified with aboved predisposing conditions in our study.

Thoracic actinomycosis, including intrathoracic organs and the thoracic wall, accounts for $15-20 \%$ of cases [1]. Actinomyces rarely causes endocarditis but sometimes can be life-threatening [22], and there are just some case reports about this uncommon type of actinomycosis [23]. Thoracic actinomycosis exhibits a pulmonary infiltrate, causing cough and hemoptysis (most common clinical signs), chest wall pain, weight loss, sputum production or draining sinuses from the chest wall and later can be disseminated to the pleura, pericardium or chest wall[24]. The clinical manifestations of pulmonary actinomycosis may be different in different regions. Hemoptysis was more commonly seen in Asian patients while chest pain were reported to be the most common complaints in Europe [25]. Our study also reveals a high incidence of hemoptysis, which is twice of chest pain. Furthermore, imaging modalities in pulmonary actinomycosis are still nonspecific and undiagnostic. Without microbiological or histological confirmation, misdiagnosis for malignancy, tuberculosis or other infections is still fairly common. FDGPET may be a proper choice to distinguish infection and malignancy for only one patient was misinterpreted as malignancy in total 4 patients undertaken the examination in our study. What's more, only two patients were confirmed by culture of bronchopulmonary secretions through flexible bronchoscopy, obviously less than that reported in Simon Bonnefond study[21]. Coexistence of actinomycosis with neoplasm or tuberculosis is seen in patient 18 and 20 , leading to a more challenging diagnosis and treatment of thoracic actinomycosis.

Risk factors for the abdominopelvic form can be abdominal operations, perforated acute appendicitis or colonic diverticulitis, mesenteric vascular insufficiency, ingestion of foreign bodies, caesarean sections or presence of prosthetic devices such as IUD contraceptives [26]. In our study, 3 female patents all has a history of prolonged use of an IUD, among whom patient 26 had the IUD for more than 20 years. It has 
been reported that the abdominal form presents classically as a slowly growing tumor, usually at the ileocecal region (less often at the stomach, duodenum, liver, rectum or several organs), causing vague abdominal pain, weight loss, low-grade fever, nausea or vomiting. For pelvic form, the most common clinical complaints are lower abdominal discomfort, abnormal vaginal bleeding or discharge [27]. But all the symptoms are often nonspecific and differential diagnosis often involves digestive and genital tumors, inflammatory disease and endometriosis. In our study, we reported an unusual case of abdominal wall actinomycosis with no risk factors which is different from other cases reported [28, 29]. Other sites of actinomycosis, such as the central nervous system, urinary tract, bones, muscle tissue and skin are rare [1].

Actinomyces spp. is known to be susceptible to penicillins and to other ß-lactams, with the exception of oxacillin, dicloxacillin, and cephalexin. This anaerobe is also susceptible to doxycycline, clindamycin, erythromycin and clarithromycin, linezolide, and tigecicline. In our study, one patient was cured by prescribing doxycycline because of allergic history of pencillin. Although the organism is multisusceptible, in most cases of infection, an initial intravenous treatment with antibiotics for 2 to 6 weeks (preferably with penicillin $\mathrm{G}$ ) with subsequent prolonged oral treatment for 6 to 12 month (amoxicillin 2g/day divided in 4 doses) is required[1]. Antibiotic treatment duration depends on infection localization and severity, and concomitant surgical approach may be required. Complete resection of the infection lesions alone is effective showed in our study but the necessity of surgery must be fully evaluated. In conclusion, individualized treatment programs based on the the best characteristics of patients is an ideal therapeutic schedule for actinomycosis.

A few limitations are apparent in this study. First, as a retrospective analysis, selection bias may have affected the statistical robustness to some extent. Second, ten patients were lost to follow-up; however, the other patients were followed at least for 9 months, which can be considered as satisfactory in the setting of an infectious disease. Third, almost all of our patients were diagnosed from histological criteria postoperatively, some cases of actinomycosis could be missing. In spite of these defects, this study provides some important information about actinomycosis in a developing country, and a comparison with that in developed countries will expedite the understanding for actinomycosis.

\section{Conclusions}

In conclusion, actinomycosis is a rare disease even in developing countries, and both misdiagnosis and missed diagnosis are common. Diagnosis was often delayed and obtained from histopathology postoperatively in developing countries. Hence, clinicians should be aware of this disease in patients with high riks factors. Complete resection of the infection lesions alone is effective and it is better to go for personalized duration of antibiotic therapy on the basis of patient disease-related parameters, especially anatomical location, rather than a fixed duration antibiotic course. In the future, we hope that specific molecular methods may help to improve early diagnosis and treatment.

\section{Abbreviations}


AMC: Amoxicillin-clavulanate; AMX: Amoxicillin; CNS: Central nervous system; CRP: C-reactive protein; CT: Computed tomography; Doxy: Doxycline; EBUS: Endobronchial ultrasound guided transbronchial needle aspiration; F: Female; FDG-PET: Fluoro-2-deoxy-D-glucose positron emission tomography/computed tomography; FV: Fever; HIV: Human immunodeficiency virus; IQR: Interquartile range; IUDs: Intrauterine devices; L: Local symptoms; M: Male; MRI: Magnetic resonance imaging; NR: Not recorded; Pen G:

Penicillin G; SUV: standard uptake value; T: Tumefaction; TZP: Piperacillin-tazobactam; WBC: White blood cell; WL: Weight loss

\section{Declarations}

\section{Acknowledgements}

Not applicable.

\section{Authors' contributions}

Study conception and design: XWX and JP. Acquisition, analysis and/or interpretation of data: $Y Y X$ and $X W X$. Drafting/revision of the work for intellectual content and context: YYX, JZ and ALL. Final approval and overall responsibility for the published work: XWX. All of the authors read and approved the final manuscript.

\section{Funding}

None.

\section{Availability of data and materials}

The datasets supporting the conclusions of this article are included within the article and its figures and tables. Additional data may be available from the corresponding author upon reasonable request.

\section{Ethics approval and consent to participate}

The study was approved by the clinical research ethics committee of Nanfang Hospital of Southern Medical University. This study was conducted in compliance with good clinical practices and the Declaration of Helsinki principles. All collected data were anonymized in standardized forms. The need for written informed consent was waived as all data were retrospectively reviewed and analysed anonymously. 
Consent for publication

Not applicable.

\section{Competing interests}

The authors declare that they have no competing interests.

\section{Author details}

${ }^{1}$ State Key Laboratory of Organ Failure Research, Guangdong Provincial Key Laboratory of Viral Hepatitis Research, Department of Hepatology Unit and Infectious Diseases, Nanfang Hospital, Southern Medical University, Guangzhou 510515, Guangdong Province, China

\section{References}

1. Wong VK, Turmezei TD, Weston VC: Actinomycosis. BMJ (Clinical research ed) 2011, 343:d6099.

2. Smego RA, Jr., Foglia G: Actinomycosis. Clinical infectious diseases : an official publication of the Infectious Diseases Society of America 1998, 26(6):1255-1261; quiz 1262-1253.

3. Xu Y, Shi J: Disseminated Actinomycosis. The New England journal of medicine 2018, 379(11):1071.

4. Paulo CO, Jordao S, Correia-Pinto J, Ferreira F, Neves I: Actinomycosis, a lurking threat: a report of 11 cases and literature review. Revista da Sociedade Brasileira de Medicina Tropical2018, 51(1):7-13.

5. Li J, Li Y, Zhou Y, Wang C, Wu B, Wan J: Actinomyces and Alimentary Tract Diseases: A Review of Its Biological Functions and Pathology. BioMed research international 2018, 2018:3820215.

6. Kononen E, Wade WG: Actinomyces and related organisms in human infections. Clinical microbiology reviews 2015, 28(2):419-442.

7. Valour F, Senechal A, Dupieux C, Karsenty J, Lustig S, Breton P, Gleizal A, Boussel L, Laurent F, Braun E et al: Actinomycosis: etiology, clinical features, diagnosis, treatment, and management. Infection and drug resistance 2014, 7:183-197.

8. Jeong YJ, Suh HW, Shim HS: Cervicofacial Primary Cutaneous Actinomycosis: Surgical Treatment for Complete Remission of the Disease. The Journal of craniofacial surgery 2017, 28(3):e269-e271.

9. Claesson R, Sjogren U, Esberg A, Brundin M, Granlund M: Actinomyces radicidentis and Actinomyces haliotis, coccoid Actinomyces species isolated from the human oral cavity. The Canadian journal of infectious diseases \& medical microbiology = Journal canadien des maladies infectieuses et de la microbiologie medicale 2017, 48:19-26. 
10. Zhang $M$, Zhang $X Y$, Chen YB: Primary pulmonary actinomycosis: a retrospective analysis of 145 cases in mainland China. The international journal of tuberculosis and lung disease : the official journal of the International Union against Tuberculosis and Lung Disease 2017, 21(7):825-831.

11. Ding X, Sun G, Fei G, Zhou X, Zhou L, Wang R: Pulmonary actinomycosis diagnosed by transbronchoscopic lung biopsy: A case report and literature review. Experimental and therapeutic medicine 2018, 16(3):2554-2558.

12. Lee MJ, Ha YE, Park HY, Lee JH, Lee YJ, Sung KS, Kang Cl, Chung DR, Song JH, Peck KR: Osteomyelitis of a long bone due to Fusobacterium nucleatum and Actinomyces meyeri in an immunocompetent adult: a case report and literature review. BMC infectious diseases 2012, 12:161.

13. Huang C, Al-Essawi T: Actinomycosis of the urinary bladder. Canadian Urological Association journal = Journal de l'Association des urologues du Canada 2013, 7(7-8):E502-504.

14. Mehrotra N, Baidya A, Brijwal M, Aggarwal R, Chaudhry R: Actinomycosis of eye: Forgotten but not uncommon. Anaerobe 2015, 35(Pt B):1-2.

15. Catano JC, Gomez Villegas SI: IMAGES IN CLINICAL MEDICINE. Cutaneous Actinomycosis. The New England journal of medicine 2016, 374(18):1773.

16. Mishra A, Prabhuraj AR, Bhat D, Nandeesh BN, Mhatre R: Intracranial Actinomycosis Manifesting as a Parenchymal Mass Lesion: A Case Report and Review of Literature. World neurosurgery 2019, 122:190-194.

17. Skuhala T, Tomasovic D, Stimac G, Ljubicic N, Orosi D, Stanimirovic A, Vukelic D: Treatment of penile actinomycosis. Dermatologic therapy 2018, 31(5):e12636.

18. Yang XX, Lin JM, Xu KJ, Wang SQ, Luo TT, Geng XX, Huang RG, Jiang N: Hepatic actinomycosis: report of one case and analysis of 32 previously reported cases. World journal of gastroenterology 2014, 20(43):16372-16376.

19. Qin J, Li W, Chen Y, Xing J: Primary urachal actinomycosis: A case report. Asian journal of surgery 2019.

20. McHugh KE, Sturgis CD: The cytopathology of Actinomyces, Nocardia, and their mimickers. 2017, 45(12):1105-1115.

21. Bonnefond S, Catroux M, Melenotte C, Karkowski L, Rolland L, Trouillier S, Raffray L: Clinical features of actinomycosis: A retrospective, multicenter study of 28 cases of miscellaneous presentations. Medicine 2016, 95(24):e3923.

22. Radu CC, Camarasan A, Podila CM, Perju-Dumbrava D: Sudden Death of a Teenager Caused by Actinomyces israelii: A Case Report. Iranian journal of public health 2018, 47(9):1413-1418.

23. Cortes CD, Urban C, Turett G: Actinomyces naeslundii: An Uncommon Cause of Endocarditis. Case reports in infectious diseases 2015, 2015:602462.

24. Su J, Liu HY, Tan XL, Ji Y, Jiang YX, Prabhakar M, Rong ZH, Zhou HW, Zhang GX: Sputum Bacterial and Fungal Dynamics during Exacerbations of Severe COPD. PloS one 2015, 10(7):e0130736. 
25. Sun XF, Wang P, Liu HR, Shi JH: A Retrospective Study of Pulmonary Actinomycosis in a Single Institution in China. Chinese medical journal 2015, 128(12):1607-1610.

26. Ketata S, Ben Mabrouk M, Derbel F, Fodha I, Sabri Y, Trabelsi A, Mtimet A, Ben Hadj Hamida M, Mazhoud J, Ben Ali A et al: [Tumoral form of abdominal actinomycosis: a retrospective case series of seven patients]. La Revue de medecine interne 2010, 31(11):735-741.

27. Choi MM, Baek JH, Lee JN, Park S, Lee WS: Clinical features of abdominopelvic actinomycosis: report of twenty cases and literature review. Yonsei medical journal 2009, 50(4):555-559.

28. Karaca B, Tarakci H, Tumer E, Calik S, Sen N, Sivrikoz ON: Primary abdominal wall actinomycosis. Hernia : the journal of hernias and abdominal wall surgery 2015, 19(6):1015-1018.

29. Kim KH, Lee JS, Cho HJ, Choi SB, Cheung DY, Kim JI, Lee IK: [A case of abdominal wall actinomycosis]. The Korean journal of gastroenterology = Taehan Sohwagi Hakhoe chi 2015, 65(4):236-240.

\section{Supplementary Files}

This is a list of supplementary files associated with this preprint. Click to download.

- Table1.pdf 